\title{
Umbilical Cord Blood-Derived Mesenchymal Stem Cells
}

National Cancer Institute

\section{Source}

National Cancer Institute. Umbilical Cord Blood-Derived Mesenchymal Stem Cells. NCI

Thesaurus. Code C82688.

Multipotent stem cells of mesenchymal origin isolated from umbilical cord blood.

Umbilical cord blood-derived mesenchymal stem cells can differentiate into a variety of cell types including fibroblasts, osteoblasts, chondrocytes, myocytes, adipocytes, and endothelial cells. 